\title{
Palabras y cosas para el Trabajo Social: sentidos y significados presentes en las estrategias de intervención utilizadas por los y las Trabajadores Sociales en la Región Metropolitana'
}

\author{
Words and artifacts for social work: perspectives and \\ meanings of intervention strategies applied by social \\ workers in Metropolitan Region
}

\begin{abstract}
Alejandra González / Lorena Pérez
Alejandra González Celis es Magister en Trabajo Social de la Universidad Católica de Chile, actualmente se desempeña como académica del Departamento de Trabajo Social de la Universidad Alberto Hurtado. Su dirección electrónica es: algonzal@uahurtado.cl. Lorena Pérez Roa es Magíster en Antropología y Desarrollo de la Universidad de Chile, actualmente se desempeña como académica del Departamento de Trabajo Social de la Universidad Alberto Hurtado. Su dirección postal es Cienfuegos 46 A, Santiago Chile su dirección electrónica es: loperez@uahurtado.cl
\end{abstract}

\begin{abstract}
Resumen
Esta investigación realizada el año 2007, buscó explorar los sentidos socioculturales que Trabajadores Sociales de la Región Metropolitana le otorgan a su quehacer, a fin de descubrir los alcances, posibilidades y límites en las estrategias de intervención utilizadas.

El proceso consideró: indagación bibliográfica para comprender los contextos políticos y de formación; construcción de categorias que podrian incidir en las estrategias; realización de doce entrevistas semiestructuradas y, reconstrucción analítica de la constelación de factores asociados a la intervención profesional en Trabajo Social.

Los resultados muestran que los profesionales vinculan linealmente espacio institucional e intervención, ya sea como limitante o generador de éxito (el poder es condicionante) y donde sus estrategias y técnicas van en un continuo desde lo asistencial (entendida en una relación directa con los sujetos) hasta aquellas potenciadoras y transformadoras (mediante la coordinación de equipos). Aún cuando el estudio no pretende generalizar, si permite cuestionar las actuales configuraciones profesionales y sus propuestas disciplinares.
\end{abstract}

Palabras clave. Sentidos culturales, Trabajo Social, estrategias de intervención.

\begin{abstract}
This research was conducted in 2007 and sought to explore the sociocultural meanings given by social workers of the Metropolitan Area to their tasks. The pourpose of this study was to observe the richness, possibilities and limitations of the professional intervention strategies that they display.

The research included a literature review to understand the political and educational contexts; the construction of categories that could affect the intervention strategies; twelve semi-structured interviews to social workers; and finally, the analysis of findings which may influence Social Wok intervention methods.

The main results show that social workers identify the institutional space as a context that singlehandedly determines their possibilities of successful intervention (the power is a condition). The strategies and techniques are developed from assistance (closer relationship with the clients) to empowerment (coordination's roles into a professional team). The research does not pretend to generalize but is a possibility to inquire the actual social work and their proposals.

Key words. Cultural perspectives, social work, intervention strategies
\end{abstract}

1 Investigación financiada por el Fondo Interno de Investigación de la Universidad Alberto Hurtado, año 2007. Se agradece a los Trabajadores Sociales que generosamente brindaron sus relatos y muy especialmente a Manuel Aris, Carolina Estay y Alvaro Herrera quienes participaron activamente en este proceso, como ayudantes de investigación. 
"Las cosas y las palabras van a separarse. El ojo será destinado a ver y sólo a ver; la oreja sólo a oír. El discurso tendrá desde luego como tarea el decir lo que es, pero no será más que lo que dice." Foucault, Las palabras y las cosas.

\section{De palabras y cosas en el Trabajo Social}

Hablamos de palabras y hablamos de cosas para el Trabajo Social. Este estudio comparte con Foucault (y de ahí la alusión a la obra del autor francés) que la modernidad establece una relación particular entre el discurso y la acción a representar, es decir, entre el significado y los significantes. Situación que llevada al plano de los propósitos de esta investigación, implica centrarse en las palabras, o "el decir" de nuestra disciplina, para reconocer el significado, o lo real a ser representado. De esta manera las palabras y las cosas en Trabajo Social, refieren a las preguntas: ¿Cuáles son las palabras que estamos utilizando los Trabajadores Sociales para describir nuestro quehacer? ¿Qué palabras usa la disciplina para referirse a ello? ¿Qué cosas intentan representar esas palabras? ¿Hablan esas palabras de cosas similares? ¿Qué mapa podríamos trazar a la luz de estos vectores?

La investigación parte de dos supuestos centrales:

- Que es necesario aportar al discurso argumentativo para mejor comprender el espacio del Trabajador Social en el Chile actual2. Tal como plantean Camelo y Cifuentes (2006: 170): "La reflexión conceptual en torno a la construcción de categorías que permitan comprender la fundamentación del Trabajo Social en la actualidad constituye el desafío de la producción de conocimientos, para trascender el marco de análisis de la práctica profesional, contextos, problemáticas, aplicación de métodos, que privilegian respuestas institucionales, programáticas". Hablamos, por ende, de superar la mera descripción del lugar profesional para contribuir a una comprensión compleja del actuar, desde la cual podamos generar conocimiento frente a los desafíos de la disciplina en el contexto nacional.

- Que es necesario generar evidencia empírica que permita conocer el tipo de intervención que los Trabajadores Sociales están ofreciendo a sus sujetos de intervención: aún cuando, es posible observar en las mallas curriculares chilenas y en las discusiones académicas establecidas ${ }^{3}$ propuestas innovadoras y discusiones que imponen al Trabajador Social desafíos contemporáneos a la luz de la emergencia de nuevos sujetos y sentidos de intervención ${ }^{4}$, aparece la sensación que en gran parte ese discurso se reproduciría por sobre otro tipo de Trabajo Social, "el real", que se establece en las instituciones y organizaciones sociales y que en variadas ocasiones no tiene un punto de encuentro con estas propuestas teóricas. ${ }^{5}$

Frente a ello se ha planteado por objetivo aportar en una primera fase a la comprensión del discurso que los Trabajadores Sociales desarrollan sobre su propio quehacer, para en una fase posterior, observar a la luz de estos discursos, los dispositivos de intervención que los Trabajadores Sociales diseñan e implementan. ${ }^{6}$

De esta manera y considerando el discurso como "una práctica analítica que opera, simultáneamente en tres dimensiones: a) el discurso en tanto texto -resultado oral o escrito de una producción discursiva-, b) el discurso como práctica discursiva enmarcada en una situación social concreta y c) el discurso como ejemplo de una práctica social, que no sólo expresa o refleja identidades, prácticas, relaciones, sino que las constituye y conforma" (Iñiguez, 2003:94), es que durante la primera fase de investigación, se realizó una indagación bibliográfica orientada a la comprensión historiográfica de

2 En tanto la producción posible de observar en las revistas y en la disminuida producción de libros disciplinares en Chile.

3 Puede observarse la riqueza de posturas y diferencias en las formas de nombrar presentes en el Congreso Internacional de Trabajo Social realizado en Chile el año 2006.

4 Pueden observar en Chile los aportes de Teresa Matus, y en Latinoamérica, Vicente de Paula Faleiros, Marilda Iamamoto, Susana Malacalza, Susana Cazzaniga, quienes han generado provocadoras propuestas para la profesión.

5 Esta llamada "sensación" las autoras han podido construirla en base a dos experiencias especificas:

a) El discurso que los profesionales Trabajadores Sociales entregan a los estudiantes y docentes a cargo de los espacios en práctica de la carrera de Trabajo Social.

b) El discurso de los profesionales Trabajadores Sociales que acuden a los distintos diplomados que ofrecemos como Departamento.

c) El discurso de los profesionales Trabajadores Sociales insertos en organismos estatales a los cuales como Departamento hemos debido asesorar mediante licitaciones de distinto tipo.

6 Investigación que esperamos realizar durante el año 2010. 
los contextos que sobre-determinaron la formación y ejercicio profesional de los Trabajadores Sociales investigados (principalmente a partir de la revisión de revistas de carácter profesional).

En esta fase fue posible reconstruir cuatro momentos profesionales en el Trabajo Social Chileno:

- 1925 - 1950: Momento fundacional, caracterizado por la valoración de la teoría social y el Trabajo Social internacional (principalmente norteamericano y europeo), valoración del Trabajo Social de caso y grupal. Levantamiento de la cuestión social. ${ }^{7}$

- 1960 - 1973: Momento comunitario, caracterizado por el desarrollo de la noción de comunidad asociada a un mismo espacio geográfico, identificación disciplinar con el sujeto social. Ebullición del fundamento político en la intervención. ${ }^{8}$

- 1973 - 1990: Período de resistencia y la pérdida del rango universitario, visibilización de un cierto binarismo profesional - el Trabajo Social de resistencia y localizado en ONGs con su consecuente consolidación del fundamento político en la intervención o el Trabajo Social institucionalizado, orientado al diseño y estructuración de la política social de dictadura vinculada a lo asistencial. ${ }^{9}$

- 1990 - 2007: Las políticas sociales y el desafío de la exclusión, posición profesional situada en el diseño, implementación y planificación ${ }^{10}$ de la política social.

Cabe señalar que el estudio no considera que estas fases describan una evolución del Trabajo Social chileno, sino que ha observado su propia historia a la luz de contextos que marcaron momentos y vectores específicos de acción que quisimos revelar. Interesa- ba observar además, si existiría alguna relación entre el momento de formación de los Trabajadores Sociales y sus decisiones estratégicas en la intervención.

Dado que la investigación se planteó desde la comprensión de los profesionales que actualmente se encuentran trabajando, es que se decidió centrarla en los tres últimos momentos identificados. Sin embargo, sí se consideraron los elementos metodológicos propios del primer momento durante la construcción de las categorías de análisis, dada su relevancia en la construcción disciplinar y, por tanto, su posible presencia en el discurso actual.

Ahora bien, la opción de trabajar con los discursos implicó tomar una serie de opciones de orden metodológico que apuntaron al desarrollo de una investigación de carácter intensiva más que representativa. De esta manera la selección de los casos en estudio fue por medio de métodos de muestreo no probabilísticos, es decir, los sujetos se seleccionaron según los objetivos analíticos. Escogimos 12 casos "paradigmáticos" de Trabajadores Sociales que ejercieran en la Región Metropolitana. La definición de "caso paradigmático" se construyó a partir de la consideración de los elementos que la bibliografía disciplinar configura como posibles relaciones entre los significados atribuidos a la noción de estrategia de intervención social y las siguientes dimensiones:

- Formación disciplinar (casa de estudio y grados académicos). ${ }^{11}$

- Espacios de desarrollo profesional. ${ }^{12}$

- Consideración de género. ${ }^{13}$

Para el desarrollo de las entrevistas se construyó una

7 Para mayor profundidad se puede referir el trabajo de Aylwin, Matus y Fortes "La reinvención de la memoria" Escuela de Trabajo Social, Pontificia Universidad Católica de Chile 2005.

8 Refiérase a los artículos producidos durante el proceso de reconceptualización chileno. Un ejemplo de ello en Ortega, Alicia. El Trabajo Social y la nueva sociedad. Universidad de Chile, 1972.

9 Véase los trabajos de Daniela Sanchez al respecto.

10 Véase los trabajos de Margarita Rozas y Nora Aquín también a modo de ejemplo en el caso latinoamericano y en el caso chileno obsérvese especialmente los publicados a partir de los años 90 (Quezada, Onetto, Aylwin, Jiménez) en la Revista de Trabajo Social de la Pontificia Universidad Católica de Chile (un número especial dedicado al tema, 1996, Nº68, por ejemplo).

11 Se incluyeron Trabajadores Sociales tanto de universidades públicas y privadas de modo de considerar la forma actual del mercado de formación existente. De la misma forma de intencionó la búsqueda de Trabajadores Sociales con grado de licenciatura, magíster y diplomado, de modo de también observar la diversidad de posgraduaciones presentes en el Trabajo Social chileno. No se consideró el grado de doctor, producto de la escasez de profesionales del Trabajo Social que en Chile detenten este grado.

12 Se incluyeron Trabajadores Sociales que pudieran dar cuenta de distintos espacios profesionales. Representan por ende espacios profesionales como: centros de salud públicos, áreas de bienestar de empresas privadas, Ongs dedicadas al trabajo comunitario, organismos colaboradores de Sename y municipalidades. Aún cuando este estudio no tiene pretensión de generalizar, debiamos dar cuenta de la diversidad de espacios profesionales que ocupan los Trabajadores Sociales.

13 Aún cuando nuestra profesión sigue concentrândose en el espacio de lo femenino, la muestra incluyó una proporción de 3 Trabajadores Sociales y 9 trabajadoras sociales de modo de también permitir incluir discursos que provinieran de géneros distintos. 


\begin{tabular}{|l|l|}
\hline Categoría & Descriptor \\
\hline Rol del Trabajador Social & Cómo nombra su cargo o los cargos que anteriormente tuvo. \\
\hline Tipo de objetivos o cambios & $\begin{array}{l}\text { Tipos de transformaciones que se pretenden alcanzar con la } \\
\text { intervención. }\end{array}$ \\
\hline Perspectiva ética - valores & Valores o las perspectivas éticas declaradas. \\
\hline Fundamentos conceptuales & $\begin{array}{l}\text { Líneas teóricas explícitamente declaradas que orientan su ejercicio } \\
\text { profesional. }\end{array}$ \\
\hline Valoración de la teoría y la investigación & Valoración y utilidad de la teoría en el ejercicio profesional. \\
\hline Noción de sujeto & Valoración y utilidad de la investigación en el ejercicio profesional. \\
\hline Contexto institucional \\
Cambios en el contexto & Denominación y problematización de los sujetos de intervención. \\
\hline Técnicas e instrumentos & $\begin{array}{l}\text { Caracterizar los elementos institucionales o contextuales que } \\
\text { influyen en intervención. }\end{array}$ \\
\hline Formación & $\begin{array}{l}\text { Nombre y descripción de los instrumentos y técnicas utilizadas en el } \\
\text { ejercicio profesional. }\end{array}$ \\
\hline & Valoración de los estudios de postgrado o educación continua. \\
\hline Trabajo multidisciplinario & $\begin{array}{l}\text { Relación de la formación continua con el espacio de desarrollo } \\
\text { laboral. }\end{array}$ \\
\hline & Relación y/o valoración del trabajo con otros profesionales. \\
\hline
\end{tabular}

pauta abierta de categorías construidas a la luz de los elementos que emergieron de la revisión bibliográfica, tal como lo muestra el siguiente esquema: Para validar el instrumento realizamos dos entrevistas que nos permitieron calibrar la intensión y dirección de las preguntas, de modo de evaluar su pertinencia en función de los objetivos de estudio. La recolección de información se realizó en un encuentro, de duración promedio de una hora y media. Se consideró el criterio de saturación de la muestra, es decir no se siguió indagando cuando los discursos emitidos por los y las entrevistadas no entregaban nuevos elementos de investigación.

A partir de la información obtenida, se organizaron temáticamente los fragmentos más significativos, los cuales fueron analizados y comentados por las autoras de este estudio. Luego se procedió a la codificación de cada uno de ellos, de acuerdo a las dimensiones extraídas del marco teórico, de manera de validar o corregir las categorías construidas. Las entrevistas fueron digitadas e ingresadas en el software QSR Nvivo. Con esta información se elaboró una matriz de contenido, de la cual se extrajeron los principales resultados. Todo el material primario se encuentra protegido, respetando los protocolos de confidencialidad asumidos para este estudio. Por ende no se especifican ni los lugares de trabajo, ni los nombres referidos en las entrevistas a fin de proteger la confidencialidad de nuestros entrevistados. La información obtenida nos permitió establecer relaciones de coincidencia y oposición, probando o refutando las hipótesis que determinamos a partir del marco teórico. Del mismo modo, algunas de las dimensiones en estudio fueron reformuladas a partir de los nuevos conceptos y categorías que emergieron a través del contraste empírico de la teoría.

\section{Análisis}

Para desarrollar el análisis hemos decidido reagrupar los hallazgos en dos grupos, el primero considerando la significación discursiva de los categorías construidas a partir de la revisión bibliográfica, y en un segundo grupo aquella que refieren a las tipologías de modelos estratégicos de intervención que pudieron surgir de la reconstrucción de lo planteado por la bibliografía y los modos en que los entrevistados nombraron su quehacer. Es importante destacar que esa tipología es una propuesta que surge como resultado de la investigación y que no se asume exhaustiva ni final, sino que permite diferenciar los discursos generando un diálogo con la teoría existente. 


\section{Cosas a la luz de las palabras y palabras a la luz de las cosas}

"Yo llego a trabajar a un patio de (), y me asignan un lugar al interior de ese patio de (), ahí está la oficina del Trabajador Social, una cosa que me pareció chocante de llegada, digamos, un Trabajador Social enterrado alli en el patio" (E7 23: 25)

Rol profesional: La pregunta por el rol se constituye a partir de dos niveles distintos de significados, uno asociado a la pregunta por el rol del Trabajo Social en las instituciones en las cuales ejercen o han ejercido y otra, desde la pregunta por el nombre que le asignan a su rol especifico. Se genera de este modo una distinción a nivel discursivo entre el rol de la profesión y el ejercicio individual de la misma.

En relación al "rol de la profesión" los discursos se movilizan en torno a quienes adscriben a la necesidad de diferenciarse de los otros profesionales de las Ciencias Sociales, en tanto alertan un "peligro" para la identidad profesional en la coexistencia de distintos profesionales en el espacio de la intervención social; y aquellos que consideran que la pregunta por el rol responde más a una necesidad de valoración profesional que disciplinar.

"Hemos pecado de dejar que otras profesiones ocupen estos espacios que nos "corresponden" o más que nos corresponden que perfectamente podríamos desarrollarlos" (E11 118:120).

"Igual pasa que al menos desde el Trabajo Social, con otras profesiones, no tanto con los psicólogos, pero en el trabajo que uno hace (...) Igual se des... se desdibujan los perfiles profesionales, entonces me cuesta tu pregunta ¿cachay? Me cuesta responderla, en términos de que yo siento que no sé si hubiese estudiado pedagogía, (...) antropología, no se si sería tan distinto a lo que hago ¿me entiende? (...) me cuesta diferenciar los límites (...) pero también me pasa que las otras profesiones no se hacen esta pregunta, el hacerse la pregunta ya es raro, como tan permanentemente preguntarse de su identidad, de su rol (...) como que: como que también es una expresión, un sentimiento de inferioridad ¿cachay?" (E5 96: 103)

Ahora bien, en relación al rol profesional que los entrevistados ejercen en sus espacios laborales, se autodescriben en roles asociados directamente a liderazgos en las instituciones utilizando verbos como: dirigir, monitorear, calificar, gestionar y/o coordinar. Se observa una atribución positiva a nivel discursivo en torno a este tipo de funciones, mientras aparece una cierta atribución de minoridad a aquellos verbos asociados a la implementación, los cuales se ubican en el espacio de lo "técnico", en el espacio del contacto directo con sujetos de intervención.
"Mi función básicamente es trabajar, yo tengo una jefatura intermedia como coordinador, tengo que trabajar con educadores que trabajan doce horas con los jóvenes en trato directo, ellos son los que están todos los días con los jóvenes (...) Entonces no hay que ser muy técnico para hablar con los jóvenes, que no implica que uno la verdad no suba el nivel de la conversación, pero si tú quieres plantear ciertas cosas no puedes pensar que estos jóvenes van a entender ciertas palabras que ellos simplemente las desconocen" (E2 57:62)

"y yo como sub-jefe me toca coordinar y acompañar el trabajo que realiza este equipo de seis personas en cada zona iya?, velar por su desempeño, velar por el cumplimiento de productos, de informes de evaluación, planificación de gestiones, informes de datos de cifras de cuantos jóvenes participan no sé donde iya?, todo eso es mi rol o sea mi rol es de gestión, de acompañamiento, capacitación, y de evaluación de equipo también poh, porque también está el desempeño profesional poh. (...) nosotros tenemos un plus: o yo tengo un plus, y la gente más cercana a los Trabajadores Sociales, más comunitario que es lo que yo conozco, no descalificando lo otro, eh tenemos ese plus que yo te decía, ser un agente integrador icachay?" (E5 23:30)

"Bueno, ehh en la actualidad soy jefe de equipo de una unidad de bienestar aqui en el hospital, y como tal, tengo la responsabilidad de... dirigir y coordinar al equipo de profesionales de esta unidad, y a un resto del personal de enfermería que trabaja en esta unidad. Ligado a ello también, hago labores especificas, del... de Trabajo Social digamos ino?, con los pacientes o usuarios, que están en esta unidad que son alrededor de veintidós, tiene que ver ehh en lo específico de esa parte social, $o$ de esos aspectos sociales, ya, digamos, satisfacer sus necesidades ehh personales, como ehh intelectuales, o ehh y otras necesidades que... ellos tienen. Pero también, ojo digamos que..no... no... ehh enmarquemos sólo como la responsabilidad social en esa tarea, digamos, esta misma responsabilidad de ser jefe de equipos, tiene que ver también con un nuevo rol que va asumiendo el Trabajo Social ehh y que tienen que ver con tareas que van más allá de lo netamente asistencial, y que tiene que ver con el desarrollo y la implementación de políticas sociales, ehh en este caso en el ámbito de la salud mental." (E7 101:112)

"En la constructora $(x x)$ yo más bien creé una labor de coordinación del bienestar, yo no era la que iba a terreno, iban otras Asistentes Sociales que dependian de mi (...) yo fui a terreno y (otros trabajos) y además coordinaba harto, en el fondo como que las hacía todas era como más integral mirado como Asistente Social, después no, sabes que uno va adaptando en la profesión que te tienes que ir desligando del... contacto con cada 
trabajador, porque (...) Que lo que pasa que (...) a ver las Asistentes Sociales que están en contacto con trabajadores, no digo que sean las menos calificadas ni mucho menos, precisamente tienes menos experiencia porque en la medida que tu vas avanzando en tu carrera vas ascendiendo un cargo y en la medida que tú vas ascendiendo un cargo tú empiezas a delegar ciertas funciones ¡te fijas!" (E4: 115-124)

"Mi rol quizás principal es el tema de coordinar las redes, la red social, estoy quizás un poquito más arriba, pero no por eso dejo de hacer lo otro y me gusta mucho hacer caso, me gusta mucho trabajar con familia, y de hecho todavía a pesar de que el tiempo no me lo permite sigo trabajando con algunas familias del XX, porque me gusta y porque siento que cuando uno está muy a nivel técnico y pierde la visión de abajo como que se pierde el foco, no sé si es como mi cable a tierra, seguir atendiendo familias y siento que lo hago muy bien ahhh" (E9 99:104)

El rol de coordinar y gestionar procesos, es asumido a nivel discursivo como un lugar que implica distancia con los sujetos de intervención, como una exigencia de los roles que "están un poquito más arriba". Se denota una tensión no resuelta entre ejercer cargos de poder organizacional y la "mirada" del Trabajador Social enfocada al reconocimiento de los sujetos y la integralidad en la intervención.

Objetivos de intervención: Tal como lo muestran las citas precedentes, los objetivos de intervención se pueden observar en un continuo que va desde aquellos denominados asistenciales, y que según el discurso de los entrevistados estaría asociado a un lugar de trabajo directo y/o implementador, hacia aquellos objetivos de tipo promocional, emancipatorios, los cuales se asocian a lugares profesionales denominados estratégicos, debido a sus mayores cuotas de poder.

"Ya... a ver, en el trabajo individual... partamos desde lo más básico, en el trabajo individual primero ehh aportar como para resolver problemas especificos que demanda la persona... como problematizar (... ) como tengo este problema, necesito que me ayude con esto, me permite ver más bien el punto de vista socioeconómico (...) ehh (... ) déjame ordenar (>2 segundos) mi objetivo sería como ese, como resolver, como sí! Es resolver!! (E3 76: 80)

"Mira, el objetivo del área comunitaria, es activar un sistema local de protección de derecho y eso uno puede interpretarlo como quiera, nosotros lo interpretamos de la brillante forma (ja), eh... un poco poder articular a todos los actores que tenían que ver con infancia en la comuna, entonces ahí estaba desde el trabajo con los mismos cabros, (hh), eh, coordinamos una radio insti- tucional de infancia, con profesores, en el ámbito de la salud" (E5 40:44).

Valoración de la teoría/valoración de la investigación: En esta misma línea, parece especialmente interesante observar que los Trabajadores Sociales entrevistados describen que habría mayor posibilidad de encontrar vinculaciones entre las teorías sociales y el quehacer profesional en aquellos cargos relacionados con mayor poder y que, por ende, permitirían construir y reconstruir diseños de intervención (políticas sociales y proyectos). Estos cargos facilitarían la dedicación a la realización de investigaciones profesionales que permitirían insumar estos quehaceres.

"Me dediqué a trabajar acá más bien en el ámbito asistencialista eh (.) con un modelo de consultorio... luego me he reincorporado al consultorio desde otro ámbito, porque ehh pasaba a ser jefe de programa, entonces yo asumía esta labor (investigación) como más directamente" (E6 45-48).

"Yo creo que si tuviera que definir una forma de intervención mía yo creo que sería esa (.) O sea claramente más bien en procesos (re)evaluativos de mejoras que incluyan eh... que le den importancia tanto a los procesos como a los resultados (.) entonces ahí hay para que se dinamice y funcione eso (.) entre gestión, instalarme" (E2 16-19).

Puede decirse entonces que los Trabajadores Sociales entrevistados podrían estar asumiendo que a mayor poder profesional en las instituciones tendrían mayor posibilidad de uso de referentes conceptuales, los cuales utilizarían para el diseño de procesos. Mientras que en aquellos espacios profesionales de mayor contingencia cotidiana, relacionados con la atención directa de sujetos esta vinculación se dificultaría (no habría tiempo para fundar teóricamente, ni para realizar algún nivel de investigación que supere los diagnósticos orientados a objetivos). "Pero definitivamente con la teoría yo siento que estoy más bien reñida... ¿Por qué? Porque en definitiva esta maquina es enorme, o sea, siempre está haciendo, haciendo, haciendo, siempre es el hacer... no queda mucho tiempo a veces como para leer... te queda tiempo para pensar con lo que tú ya sabes" (E3 67-70).

Ética de la intervención: En términos éticos aparecieron tres posiciones posibles de categorizar. Por un lado aquellas relacionadas a una ética fundada en los derechos humanos y el resguardo de la dignidad de la persona, en segundo lugar una ética que podríamos llamar "ciudadanizante" donde aparecían nociones como participación y democracia como principios orientadores de las estrategias a utilizar, y finalmente una ética relacionada al configurar un desempeño profesional eficiente y efectivo. 
"El tema de la perspectiva de derecho es central en el trabajo que nosotros hacemos tanto para pensar el derecho que tiene el joven, el adolescente o el niño, como para pensar en el derecho que tienen el trabajador frente a la labor que le es encomendada. Eso como principio central, no obstante a eso hay un principio desde mi perspectiva y que va a estar siempre orientado a las intervenciones que yo hago y tiene que ver con la justicia social. Desde ahi cualquier intervención que a mí me mueva, me conmueva tiene que ver justamente con la justicia social" (E5 67- 72).

"Yo creo que aquí lo que se juega son principios éticos y políticos, y por lo menos a mí, en mí yo veo mi trayectoria, que es lo que me ha movido, que me ha hecho pensar en que hace es eh (...) (. ) a ver, el tema de la toma de conciencia o (...) puede haber sido mucho más fuerte, el tema de la participación, ah? Es decir en qué medida el tema de los sujetos que se involucran en la intervención, se constituyen como sujetos y actores de su intervención" (E8 4-8).

"El compromiso como un valor importante, hem... la excelencia y la innovación, esas son como las cosas que debieran llevar la intervención como adquiriendo el compromiso que tú adquieres con las consecuencias que ello implican positivas o negativas de las cosas" (E4 87-89).

Cabe señalar que a nivel de discurso todos los entrevistados relacionan las estrategias de intervención con sus respectivos posicionamientos éticos. Es decir, reconocen en su intervención posiciones personales más que profesionales, las que en muchas ocasiones entrarían en tensión con los objetivos orientadores de sus respectivas instituciones.

Contextos institucionales/Cambios en el contexto: Si bien originalmente nuestra construcción de categorías había considerado la categoría "contexto institucional" y "cambios en el contexto" como diferentes, a nivel de discurso ambas aparecieron absolutamente relacionadas, puesto que los Trabajadores Sociales entrevistados declaran que su profesión está delimitada por el contexto institucional, el que se explica y determina por un contexto sociopolítico.

"Como programa yo creo que el noventa por ciento está financiado por el municipio, por lo tanto somos una dependencia municipal, por lo tanto aplicamos, aplicamos esa política, la política definida desde la alcaldesa ya, y mucha ingerencia en eso tampoco tenemos, más allá de lo que podemos demostrar con resultados" (E9 73-76).

"En las instituciones entre más cargay al sujeto, puede ser conflicto con la institución, si tú no compartes claramente la visión del problema que tiene la institución, también es un tema, (...) jodido, si tú no tienes resulta- dos en tu acción, también te pones en riesgo ite fijas? Es decir, siempre yo diría que, que (2 seg.) en ese sentido como que los Trabajadores Sociales, siempre pueden ser como más conflictivos im? son más críticos de las instituciones" (E10 28-32).

Es justamente la determinación de la intervención por el espacio institucional una de las principales tensiones compartidas por todos los entrevistados y en la cual descansan sus mayores frustraciones, en tanto la definición de resultados o metas fijadas por la instituciones no necesariamente se condicen con los procesos que los entrevistados plantean desarrollar.

Sin embargo, llama la atención cómo esta tensión a nivel discursivo se resuelve asumiendo un rol de poder al interior de la institución, lo que permitiría a nivel simbólico, ampliar los márgenes que limitan la intervención.

Fundamentos conceptuales: A través de esta categoría buscábamos que los entrevistados explicitaran las posturas teóricas que orientan su intervención y que le otorgan un horizonte de sentido. Sin embargo, no fue posible visibilizar corrientes teóricas específicas ni posicionamientos conceptuales claros, declarando utilizar una multiplicidad de conceptos, de los cuales los más reiterados fueron: ciudadanía, empoderamiento, pero sin producir una definición mayor.

"Mira, yo creo que el, el, el...la metodología que se utiliza $o, o, o .$. la teoría que va detrás van muy apuntados a lo que tú, a lo que tú, a donde tú te formaste y así que por ejemplo mi universidad, en ese tiempo no sé ahora, trabajaba más con enfoques más comunitarios, de educación comunitaria y dentro de esa misma dinámica que te produce, que eso fue lo que yo apliqué en una primera instancia y después aplicamos mucho la teoría sistémica $y$ de ahí desarrollábamos, $y$ de ahí fundamentábamos todos los procesos que queríamos generar, y desde ahi también evaluábamos, ya" (E9 39-45).

Cabe señalar que a nivel discursivo se plantea una confusión de niveles en relación a los enfoques teóricos y metodológicos, en tanto no resulta evidente en los discursos la vinculación y determinación de los mismos, sino más bien se plantean metodologías como teorías.

Por otro lado, resulta relevante remirar la atribución positiva, que a nivel de discurso, se le otorga a la combinación de teorías a partir de los fenómenos de intervención. Los entrevistados asumen que la realidad con la que trabajan es compleja y frente a ello dicen que es necesario utilizar todo lo que conocen, sin por ello creer que esto podría acarrear alguna incoherencia conceptual. 
Noción de sujeto: Aparecieron una variedad interesante de denominaciones las que a su vez se relacionaban con distintas posibilidades estratégicas de intervención. Fue posible encontrar en el discurso de los entrevistados nociones como: sujeto deficitario, sujeto participativo, sujeto de derecho (y su consiguiente tensión con la visión de objeto de política social), sujeto activo, sujeto receptor de servicios (cliente).

"Hemos tratado de dejar fuera prácticas que están dentro del área asistencial porque creemos en los sujetos mucho más activos, muchos más responsables de su futuro, darle alternativas y que ellos solucionen, creemos más en el sujeto más que en el objeto de atención” (E9 87-89).

"Más que conceptualización en ese tiempo (hh) hablábamos de jóvenes de sectores populares o en riesgo social" (E11 6-7)

"En realidad en el ámbito de salud, se habla más bien de usuario... ahora (...) aunque todavía no logramos pasar traspasar la... la... digamos la muralla del paciente (...) hoy en día se está hablando de usuario" (E3 50-53).

"Independiente del marco institucional yo he asumido a los sujetos como clientes (...) eh puede ser mi formación de la escuela lo que pasó es que me hizo sentido como a mi me gusta la linea de gestión yo asumo que un cliente es a quien eh quien está en condiciones de demandar el mejor servicio" (E1 36-39).

Los matices y enfoques que explican las distinciones en las nociones de sujetos de los entrevistados nos resultan particularmente interesantes, en tanto permitirían intencionar la búsqueda de posibles coherencias o incoherencias en los discursos con las estrategias de intervención que pueden plantearse. ¿Cómo empoderar a un sujeto que se considera deficitario, por ejemplo?

Técnicas e instrumentos de intervención: En este punto, los entrevistados mencionaron técnicas e instrumentos sistematizados en la bibliografía existente y utilizados transversalmente durante la historia del Trabajo Social. Se mencionó: informes de sistematización, diagnósticos, entrevistas, talleres (sumamente mencionados: talleres socioeducativos, talleres de formación, capacitaciones) y visitas domiciliarias.

"Principalmente zel trabajo de acá es hacer, son los talleres (...) sí, y charlas de discusión acerca de los derechos del niño" (E5 69-70).

"Visitas domiciliarias siempre se ha hecho dependiendo de la necesidad del caso, pero eso era básicamente el trabajo de talleres y de CASO" (E3 37-38).

"La entrevista (...) la entrevista mucho eh... eh... (...) bueno la visita domiciliaria fuerte eh... y ade- más la visita domiciliaria requería el manejo de una serie de otras técnicas más bien comunicacionales, de relaciones, de empatía eh... de adaptación" (E1 24-26).

A nivel de discurso se revela una asimetría en relación a los objetivos de intervención, la noción de sujetos y las técnicas, puesto que se observó dificultad al hablar e intentar dotarle contenido a los instrumentos de intervención que están utilizando. Se observa que, independiente del objetivo a transformar, el medio para hacerlo se limita a multiplicidad de talleres, entrevistas y visitas. O en su defecto, que se hagan "cosas", al decir Foucaltiano, que no se saben cómo nombrar rotulando de taller, entrevista y visita todo aquello que no se puede decir.

Más allá de la indudable potencia de dichas técnicas nos llama la atención que no se mencione en el discurso otro tipo de instrumentos. De hecho al observar las entrevistas en detalle, el discurso de los Trabajadores Sociales se acorta, situación radicalmente diferente a cuando se refieren a los sujetos de intervención de quienes parecen tener mucho que decir.

Trabajo interdisciplinario: Este fue un tema donde se pudo observar un discurso discordante entre los Trabajadores Sociales: por un lado se valoraba y reconocía, al trabajo interdisciplinario, como un espacio que permitía la complejización de los procesos de intervención y por ende, su discusión a la luz de distintas disciplinas, que enriquecía el quehacer y por otro lado, se mencionaba a éste como un obstaculizador para el ejercicio profesional, ya que se mencionaba una cierta competencia entre disciplinas que podrían estar teniendo un accionar similar.

"Tener un énfasis en la capacidad de trabajar en equipo, no trabajar solo, porque yo creo que de repente también nos creemos los perfectos interventores y las sabemos todas, y nos olvidamos que tenemos compañeros de trabajo" (E11 120-123).

"Los psicólogos han ido incorporándose de manera reciente a la atención primaria (.) de hecho nos llevan quitando varios espacios en la atención" (E3 28-29).

Sin duda alguna, los temores asociados al trabajo con otros profesionales nos estarían hablando de un Trabajo Social reducido a la operación de ciertas técnicas y diluido por la presencia de otros profesionales de las Ciencias Sociales que dicen hacer "lo mismo". Esto parece vital, puesto que permite preguntarse si acaso no se reducirá la noción de estrategia de intervención a su operacionalización en 
tanto técnicas, o si el eclecticismo teórico no contribuye a esa posible "difusión" profesional. Cuestión que sigue apareciendo como importante de seguir indagando.

Formación profesional: Finalmente respecto a la formación, hay una valoración positiva de ésta en tanto permite complejizar diagnósticos y comprender fenómenos. Sin embargo no aparece con fuerza, en el discurso de los Trabajadores Sociales, que esta formación impacte en términos de los rangos y/o ofertas de estrategias de intervención que ellos podrían estar diseñando e implementando, sino más bien el énfasis está en la oportunidad que les genera de acceder a nuevos espacios laborales y en el cómo observar a sus sujetos de intervención, tal como lo indican las citas precedentes:

"El magister completamente! O sea si no fuera por el magister yo no tendría el cargo que hoy tendría" (E4 29-30).

"Yo creo que si no hubiera sido por el magister yo me habría dormido en los laureles y no estaría donde estoy" (E3 50).

"Después tuve la posibilidad de hacer un magister ¿cachay? en Ciencias Sociales y yo te diría que me aportó un poquito más iya?. No he terminado mi Tesis entre paréntesis, estoy como cinco años atrasao, tengo que retomarla pero bueno (ja). Y después hice un diplomado ¿si?, un diplomado de intervención en mundos juveniles, y ese me enriqueció pero caleta, porque mi tecla ha sido todos estos años el mundo juvenil, desde lo popular" (E11 112 - 116).

\section{Lugares para palabras y cosas}

A partir de una primera interpretación del quehacer profesional de cada uno de los contenidos que hemos expuesto, nos fue posible como investigadoras levantar una suerte de categorización donde podríamos ubicar las distintas "cosas" que los Trabajadores Sociales dijeron realizar, sobredeterminados por su rol profesional, objetivos de intervención, valoración de la teoría/valoración de la investigación, ética de la intervención, contextos institucionales/ cambios en el contexto, fundamentos conceptuales, noción de sujeto, trabajo interdisciplinario y formación profesional.

Decidimos utilizar la noción de estrategia y no la de metodologías o técnicas en base a lo que Faleiros (2003:75) plantea: "Las estrategias son procesos de articulación y mediación de poderes y cambio de relaciones de intereses, referencias y patrimonios en juego, ya sea por la recomposición de recursos, de ventajas y patrimonios personales, ya sea por la efectivización de derechos, de nuevas relaciones o por el uso de informaciones. Las estrategias implican inversiones en proyectos individuales y colectivos que aporte rearticulación de patrimonios, referencia e intereses con vistas a la re - producción y a la re presentación de los sujetos históricos. Reproducirse es atender a las necesidades de supervivencia en las relaciones sociales dadas históricamente y re - presentarse significa el proceso de re - construcción de la identidad." Es decir, observamos que en la noción de estrategia se articularían los distintos elementos que forman parte de la constelación profesional, a la luz del proceso de transformación que se pretende llevar a cabo. Utilizamos dicha noción en la construcción de las categorías que constituyen el cuerpo de esta investigación, sin embargo, no representan linealmente el habla de los sujetos entrevistados. Hemos interpretado ese discurso en base a esta noción de estrategia.

Es importante reafirmar que esta categorización, vale decir, los tipos de estrategias que a continuación se describen no intentan ser exhaustivas ni comparables en la totalidad de sus dimensiones. Probablemente ese sea una tarea pendiente a realizar a la luz de estos resultados, en tanto no necesariamente los lugares mapeados comparten nivel o tipologías que la bibliografía declara existir en la intervención profesional.

Pareció importante respetar las aparentes contradicciones discursivas, ya que precisamente es esa contradicción la que nos daría ciertas luces sobre el tipo de intervención que los Trabajadores Sociales realizan, la cual no necesariamente es lineal.

Estrategias Individuales: Relacionadas al Trabajo Social de caso o fundacionalmente llamado case work. Trabajo Social que podría tener contenido terapéutico (dependiendo de la necesidad del sujeto que se encuentra en frente) y que funciona en base a la relación uno a uno (profesional / sujeto). Discursivamente para los Trabajadores Sociales entrevistados tiene un polo relacionado con lo asistencial y cortoplacista mientras en el polo opuesto, se orientaría por objetivos de desarrollo personal e incluso reparatorios de los sujetos. Sin embargo sea cual fuere el polo, aparecería vinculado a espacios de minoridad de poder, vale decir, al trato directo, al trabajo emergente, urgente y tensionado -vastedad de usuarios, variedad de demandas-.Tal como se puede observar en las siguientes citas:

"Lo que pasa es que aquí hay un modelo, o sea la idea es que cambie, pero siempre dentro de las instituciones de salud la asistente social es como la que resuelve todo lo que el resto no puede resolver, es como es que este caso no lo puedo... me complica, te lo mando a ti (...) es como 
mucho eso (...) y además ehh la comuna en que estoy yo es como bien em (.) a ver ¿Como decirlo? bueno asistencialista, o sea esta cosa como de resolver el problema concreto de la persona sin mirar más allá, o sea acá hay mucho de atención de caso" (E3: 45-50).

"Te llegaba el caso revisabas la carpeta entonces (se realizaban) las visitas domiciliarias o entrevistas que eran necesarias y evacuabas el informe y venía el siguiente (...) y a veces volvías a ver a la familia porque los que llegaban por alimentos, después llegaban por visita, después llegaban por tuición y después entonces pasaban por todas las medidas (...) los Trabajadores Sociales que estábamos ahí generalmente una de las cosas que se quejaban era que no se podia hacer intervención en el fondo" (E2 92:97).

Es interesante observar esto a la luz de lo planteado por Du Ranquet (1996:XIV) al decir: "Quizás como sostiene Payne (1995), sin estar en el horizonte un cambio de paradigma, pues aunque aparezcan importantes cambios en las teorías y en las prácticas, las Trabajadoras y Trabajadores Sociales conservan un modelo de explicación aún muy influenciado por la psicología dinámica y un modelo de intervención muy condicionado por el Trabajo de Caso Social Individual (social casework)", situación que podríamos estar observando en la forma en que se configura este tipo de estrategia.

Estrategias grupales: Estrategias que se focalizan en sujetos grupales: jóvenes, niños, pandillas, adultos mayores. Parte de la base de una tipología común, y desde allí postula actividades y técnicas grupales que permiten a los sujetos, en su reconocimiento como categorías, superar la individualidad y por tanto, sus problemáticas. Estrategias que se podrían implementar cuando se tiene una cuota mayor de poder -mayor que el de las estrategias clínicas-, pero que no se relaciona con un espacio de poder institucional en tanto sus sujetos de intervención son determinados por contextos institucionales superiores.

Aquí la única técnica de intervención que aparece en el discurso de los Trabajadores Sociales es el taller, sin mayor especificidad del modo en el cual éste se configura:

"Entonces nosotros a ellos los convocábamos (...) eh: mira primero el trabajo grupal es una de las estrategias que conocí, aprendi desde las metodologías participativas, mucha educación popular iya? Y (...) sin darme cuenta, con el tiempo lo supe mucho construccionismo, construir la intervención grupal y la realidad grupal, desde la participación de todos los integrantes iya?" (E11 35- 38).

"Haciamos talleres para las madres de niños, de niños desnutridos, eh talleres de formación, sobre nutrición, sobre cosas muy básicas, sobre cosas muy básicas y básicamente era un trabajo de casa a casa, a través, de cruzábamos largas distancias por que El Monte no es una ci, es ciudad, pero también tiene mucho ámbito rural dentro de eso. (...) talleres de formación, talleres socio-educativos y talleres de formación a personas que trabajaban con, con, con que trabajaban con estas madres, como monitoras de salud" (E6 68:73).

"Aquí esto tenía que ver más con un tema grupal, de poder trabajar con las personas que estaban con consumo de alcohol, que ellos pudieran ver que habían otras posibilidades, de otra gente que también estaba en en el mismo proceso, y poder enriquecerse de eso y junto con ellos llevábamos un grupo también de familiares, y ahí venían mucho más las mujeres que ellos, y eso era para ver destrezas específicas que ellos como que tenían que poner... o sea trabajar, para poner en práctica en definitiva, para mantener la conducta del no consumo... y evitar recaídas... eso... emm" (E3 65-71).

Estrategias de Resolución de conflictos: Según la bibliografía existente, también sería posible encontrar al Trabajo Social en un rol de mediador, de interfaz, entre sujetos y entre los sujetos y las instituciones. Habría utilización de variadas técnicas -contratos, debates, etc.-Estas estrategias se realizan con sujetos individuales, grupales y comunitarios -mediación familiar, comunitaria, etc.-. Los Trabajadores Sociales pueden mencionar y relatar a la mediación como un lugar distinto de intervención, y probablemente ello tenga relación con el impacto que ha tenido como discurso en nuestra profesión y en otras ${ }^{14}$. Según el discurso de los Trabajadores Sociales entrevistados, quien realiza mediación es un sujeto con estudios y por ende detenta mayor poder y posibilidad de acción y reflexión.

"Bueno; también se hace mediación, mediación entre los miembros de las familias, igual hay parte del equipo que está capacitado en eso, tienen un diplomado" (E5 43:44).

Estrategias de Desarrollo Local: El Trabajador Social en un rol de promotor social, articulador de actores y procesos en los espacios locales. Aquí, según el discurso investigado, es posible evidenciar poder y utilización de teorías e investigación. El profesional investiga, reconoce recursos, dirige y

14 Baste observar cómo el mediador existe como figura en la nueva justicia chilena. 
coordina. Aparece como una suerte de "lugar ideal" de intervención donde el Trabajador Social podría liberarse de dominios institucionales. Sin embargo, escasamente se reconocen a sí mismos como potenciadores de este tipo de estrategias, ya que parece en el discurso un Trabajo Social que pudo haberse realizado antes.

"Era el trabajo organizacional, porque los asentamientos tenían una organización, era una organización con una directiva y con diferentes comités, entonces nuestra función era ehm, en ese ámbito era, ehm, orientar, orientarlos acerca del cumplimiento de sus funciones dentro de la organización, hacíamos también trabajo con mujeres, con niños ehh, en el ámbito comunitario (...) la otra dimensión organización comunitaria o vecinales de jóvenes iya? Que es distinta a la institucional, porque en una están los profesionales que intervienen en temáticas en la otra están los mismos jóvenes" (E11 105-108/120-123).

"En ese consultorio, el consultorio XX fue, un trabajo de desarrollo social, de desarrollo local, donde trabajé, donde pensamos, donde creamos icierto? La creación.... pensamos la creación de comités de salud, comités locales de salud, comités comunales de salud, fue en Quinta Normal donde se inicia ese trabajo, un poco de recrear, de repensando los antiguos comités de salud, dijéramos en la época de los años setenta, eh repensando esto, y haciéndolo más en la actualidad no, en la idea de que la gente participara en la gestión de la salud... en su comunidad" (E6 86-92).

Estrategias de Redes Sociales: El Trabajador Social como un ente provocador de sinergias que superan lo local -redes supralocales, interinstitucionales-; movilizador de recursos. Ubicado en un rol de poder y en un espacio estratégico que le permite relacionarse con distintos tipos de profesionales e instituciones y/o organizaciones. En rol que "coordina" a otros que trabajan directamente con los sujetos. Tal como plantea Acosta (2000:15): "Participar, formar parte de redes sociales, operar desde las mismas no se limita a un cambio de concepciones y valoraciones, sino que exige una praxis de transformación mediante formas organizativas democráticas que generen cambios en la estructura de poder y en la gestión política del Estado. Cambios que se gestan, se construyen en el propio seno de las relaciones sociales".

"Mi rol quizás principal es el tema de coordinar las redes, la red social, estoy quizás un poquito más arriba" (E9 99-100).

"Es decir, yo siempre he sido más como de la tendencia de trabajar los ámbitos colectivo que los ámbitos individuales $m$ ? o que el ámbito individual que no se resuelve por yo aislarme y buscar una solución a mi problema, si no, por el contrario mi (...) mi situación de problema que yo ah? Cuestión de derechos humanos ah? o temas de salud, o temas de educación, o temas institucionales, se resuelve en la medida en que... si trabajamos en conjunto, trabajamos en redes, en asociatividad" (E10 57-62).

"Y desde esa perspectiva es conocer todas las entidades no solo municipales, si no también redes locales, como ONG's y entidades que están instaladas dentro de la comuna y en ese ámbito, trabajar colaborativamente, que es lo que efectivamente funciona" (E10 23-25).

Estrategias de Empoderamiento o Desarrollo de Capital Social: El Trabajador Social como actor político que puede entregar poder, que puede dirigir y manipular flujos de capitales sociales, y que por ende puede levantar nuevas categorías. Se mencionan aquí procesos de intervención que ya no tienen directa relación con sujetos sociales. El Trabajador Social levanta y promueve, coordina la implementación, empodera equipos y profesionales.

"Las acciones concretas pueden ser:: organizarse, las acciones concretas pueden ser eh:: tomar liderazgo de un grupo eh::, las acciones concretas pueden ser hacer algo en conjunto para resolver tal o cual cuestión, eh:: es decir, pero siempre con un foco más de la acción colectiva ah? (.), en vez del beneficio individual, es decir, si uno quiere que los sujetos tomen conciencia, y sean partícipes ah? Tienen que eh: (.) generar acciones pequeñas o más grandes, o más puntuales, (.) más generales, en torno a esa situaciones (3) ehm" (E8 116:122).

"Empoderar a la red de infancia, que los actores locales se hagan cargo de su responsabilidad en el sistema de protección local, ese yo creo que ha sido mi trabajo, que es un trabajo con una dimensión simbólica" (E5 85-88).

Estrategias de Planificación Social: El Trabajador Social como diseñador y evaluador de la política social. Como constructor de categorías, como investigador -es interesante como los Trabajadores Sociales asumen que solo desde un lugar de planificación pueden realizar investigaciones-. Trabajadores Sociales situados en cargos de reconocido poder son, según los entrevistados, quienes pueden implementar estas estrategias, lugares que por cierto, son escasos.

"Por lo tanto acá la asistente social, el rol básicamente que tenía era de levantar el informe de una realidad en un momento determinado, de un corte de esta realidad a la que ella llegaba, a veces muchas veces desconociendo lo anterior y lo posterior, y lo que en ese momento tú te encontrabas, por lo tanto muy parcial, con muy pocas posibilidades de hacer algo, o nada, no era lo que yo necesitaba, o lo que yo quería (...) aquí me toca coordinar 
una serie de programas que están en forma transversal, me toca planificar" (E10 46-50).

"Bueno, ehh en la actualidad soy jefe de equipo de una unidad de bienestar aqui en el hospital, y como tal, tengo la responsabilidad de... dirigir y coordinar al equipo de profesionales de esta unidad, y a un resto del personal de enfermería que trabaja en esta unidad. Ligado a ello también, hago labores específicas, de el... de Trabajo Social digamos ino?, con los pacientes o usuarios, que están en esta unidad que son alrededor de veintidós, tiene que ver ehh en lo especifico de esa parte social, o de esos aspectos sociales, ya, digamos, satisfacer sus necesidades ehh personales, como ehh intelectuales, o ehh y otras necesidades que... ellos tienen. Pero también, ojo digamos que..no... no... ehh enmarquemos sólo como la responsabilidad social en esa tarea, digamos, esta misma responsabilidad de ser jefe de equipos, tiene que ver también con un nuevo rol que va asumiendo el Trabajo Social ehh y que tienen que ver con tareas que va más allá de lo netamente asistencial, y que tiene que ver con el desarrollo y la implementación de políticas sociales, ehh en este caso en el ámbito de la salud mental." (E7 101:112).

\section{Conclusiones}

La preocupación por el rol del Trabajo Social ha sido sistemática en el discurso latinoamericano. Marcar un horizonte y/o debatir sobre lo que se quiere de la profesión es temática recurrente de congresos y encuentros, sin embargo, parece desconocerse la configuración disciplinar que el Trabajo Social podría estar asumiendo en nuestros días.

Esta investigación levantó cuatro momentos profesionales, sin embargo a la hora de observar el discurso de los Trabajadores Sociales entrevistados, no fue posible generar una correlación entre momentos y tipos de estrategias utilizadas, en tanto los profesionales de manera transversal plantearon la determinación de las estrategias y técnicas de intervención utilizadas por las instituciones en las cuales se desenvuelven. Asumiendo que se les entrega un rol y desde ese rol se realiza la intervención.

A nuestro modo de ver esto representa un hallazgo necesario de ser remirado en tanto hablamos aquí del discurso de 12 casos paradigmáticos provenientes de distintas casas de estudio y periodos de formación, sin embargo el sentido parece ser coincidente. ¿Formará parte ello de una tendencia en Chile?

Por otro lado, se reconocen un sinnúmero de desafíos en torno a las significaciones que estos Trabajadores Sociales exponen en sus discursos y las implicancias de ello en sus estrategias de intervención social. Si bien, esta investigación sólo pretende ser una primera aproximación a los discursos, se abren preguntas extremadamente sensibles para la profesión y para los procesos de intervención profesional, tales como: ¿Qué implicancias puede y/o está teniendo, el hecho que los profesionales consideren en una ubicación de minoridad aquellas estrategias relacionadas con la intervención directa con sus sujetos de intervención? O por el contrario, ¿por qué el rol de coordinador, director, diseñador de la política, pareciera ser "el" espacio de desarrollo fundado y con poder? ¿Qué impacto tiene esto en los cambios sociales hacia los cuales los Trabajadores Sociales intentan aportar (objetivos, propuestas éticas, etc)?

A través de esta investigación hemos buscado abrir un espacio que permita aportar y comprender el estado de situación profesional en relación a lo que hacemos y al nombre y lugar en que lo situamos. Con ello hemos querido levantar discursos y provocar preguntas más que proponer interpretaciones.

Durante el año 2008 tuvimos la oportunidad de compartir nuestros resultados de investigación en el congreso de Trabajo Social realizado en la ciudad de Montevideo, y es evidente que el contexto sociopolítico chileno ha impactado sobre nuestra disciplina haciendo que el perfil tecnocrático conviva con profesionales que siguen aspirando a aportar a procesos de transformación social. Ello abre, a nuestro parecer, una dualidad entre el tipo de Trabajo Social que se aspira a hacer y del que se habla y el tipo de Trabajo Social que se hace y que se silencia.

La fuerza del discurso sobre los sujetos, la convicción en cada uno de los entrevistados de que el nombrar contiene una forma de entender al otro, pareciera hablar de un compromiso profesional con el sujeto claro y fundamentado, sin embargo dicho compromiso se desvanece, a nivel discursivo, cuando hablamos de estrategia. ¿Por qué la coherencia en el discurso se "fija" en las tipologías de sujeto? ¿Por qué es de los sujetos de lo que más puede hablarse, mientras que se asume que es precisamente el lugar de lejanía con el sujeto el que entrega poder de transformación de realidad? ¿Qué implica ello en un contexto institucional que se asume como limitante e intransformable? ¿Cómo se convive en la cotidianeidad de la intervención con este tipo de discursos?

A nuestro parecer: Los Trabajadores Sociales que compartieron su discurso se apasionan enormemente cuando hablan de sus sujetos de intervención, se 
frustran cuando hablan de su espacio institucional. Lo que podría interpretarse como un intento por mostrar que se encuentran en un rol de poder cuando no están con esos mismos sujetos que tanto les apasionan. Sin embargo, asumen en otros momentos que cuando están con sus sujetos, otros profesionales también podrían estar desarrollando el mismo trabajo. ¿Qué efectos tiene ello en la intervención que ese profesional puede ofertar? Es precisamente en esa línea que esperamos poder seguir desarrollando investigación.

\section{Referencias}

ACOSTA, BLANCA. (2000): Redes sociales: una estrategia de gestión comunitaria, en Revista regional de Trabajo Social del Uruguay, № 20.

AYLWIN, MATUS, FORTES. (2005): La reinvención de la memoria. Escuela de Trabajo Social, Pontificia Universidad Católica de Chile.

CAMELO, \& CIFUENTES. (2006): Aportes para la fundamentación de la intervención profesional en Trabajo Social en "Tendencias y retos" Revista de la Facultad de Trabajo Social Universidad de La Salle, $N^{\circ} 11$.

DIVERSOS AUTORES. (1996). Revista de Trabajo Social de la Pontificia Universidad Católica de Chile, Nº68.

DU RANQUET, MATILDE. (1996): Los modelos en Trabajo Social, Intervención con personas y familias, Madrid: Editorial Siglo Veintiuno.

FOUCAULT, MICHEL. (ed 2002): Las palabras y las cosas, Madrid: Editorial Siglo Veintiuno.

IAMAMOTO, MARILDA. (2003): El servicio social en la contemporaneidad, Buenos Aires: Cortez Editora.

IÑIGUEZ, LUPICINO. (2003): Manual de análisis de discurso para las Ciencia Sociales. Barcelona UOC.

MALACALZA SUSANA. (2007): Hilos y nudos la formación, la intervención y lo político en Trabajo Social. Buenos Aires: Espacio.

MATUS, TERESA. (1999): Propuestas contemporáneas en Trabajo Social: hacia una intervención polifónica. Buenos Aires: Espacio.

PÉREZ, GLORIA. (1994): Investigación Cualitativa: Retos e Interrogantes. Editorial La Muralla, España. Volumen I.

POTTER, JONATHAN. (1998): La representación de la realidad: Discurso, retórica y construcción social. Editorial Paidós. 